\title{
Tiling a strip with triangles
}

\author{
John Bodeen Steve Butler Taekyoung Kim \\ Xiyuan Sun Shenzhi Wang \\ Department of Mathematics \\ Iowa State University \\ Ames, IA, U.S.A. \\ \{jbodeen, butler, shawnkim, xiyuansu, shenzhiw\}@iastate.edu \\ Submitted: Jun 18, 2013; Accepted: Nov 30, 2013; Published: Jan 12, 2014 \\ Mathematics Subject Classifications: 11B39, 52C20
}

\begin{abstract}
In this paper, we introduce the tilings of a $2 \times n$ "triangular strip" with triangles. These tilings have connections with Fibonacci numbers, Pell numbers, and other known sequences. We derive several different recurrences, establish some properties of these numbers, and give a refined count for these tilings (i.e., by the number and type of triangles used) and establish several properties of these refined counts.
\end{abstract}

Keywords: triangle tilings; Fibonacci numbers; Pell numbers

\section{Tiling with the triangular strip}

A well known combinatorial interpretation of the Fibonacci numbers (i.e., $F(0)=0$, $F(1)=1$, and $F(n)=F(n-1)+F(n-2))$ is the tiling of a $2 \times(n-1)$ rectangular strip with vertical and horizontal dominoes. For example $F(5)=5$ and there are 5 tilings of the $2 \times 4$ strip:
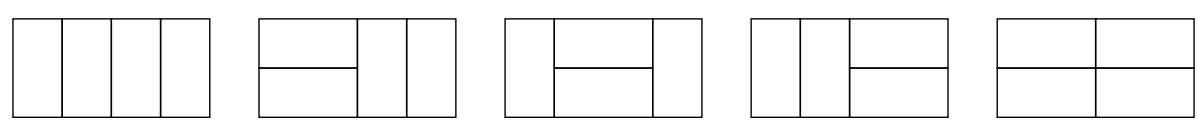

This interpretation can be used to give combinatorial proofs of many interesting properties of the Fibonacci numbers (see [1]).

We want to explore a variation of tiling on a board which shares many similar properties. Namely we want to consider tiling a $2 \times n$ "triangular strip". This consists of a parallelogram constructed from $4 n$ equilateral triangles which has two rows of $2 n$ triangles. As an example, the $2 \times 6$ triangular strip is shown below. 


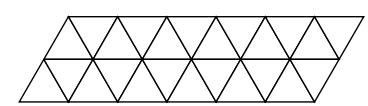

There are four types of triangles available for us to use for tiling this strip, i.e.,

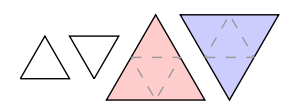

which we will denote as $1^{+}, 1^{-}, 2^{+}$, and $2^{-}$respectively, the latter two of which we will sometimes call the large triangles.

Observation 1. The number of ways to tile the $2 \times n$ triangular strip using triangles of type $1^{+}, 1^{-}$, and $2^{+}$is $F(n+1)$, i.e., the same as in tiling the rectangular strip. By rotating the board $180^{\circ}$ the same result holds for tiling with triangles of type $1^{+}, 1^{-}$, and $2^{-}$.

As an example the five ways to tile the $2 \times 4$ triangular strip are shown below. The observation follows by noting that in both cases the tiling can be determined by reading along the bottom edge.

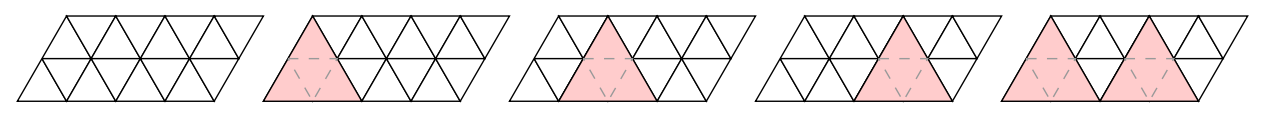

We will use this several times to connect our tilings with Fibonacci numbers. In general we are interested in the number of tilings which use all four of the types of triangles. We first determine a recursion formula that these tilings satisfy.

Theorem 2. Let $I(n)$ denote the number of tilings of the $2 \times n$ triangular strip using triangles of type $1^{+}, 1^{-}, 2^{+}$, and $2^{-}$. Then $I(1)=1, I(2)=4, I(3)=8$ and for $n \geqslant 4$

$$
I(n)=I(n-1)+3 I(n-2)+I(n-3) .
$$

Proof. The tilings for $n=1,2,3$ are as follows:

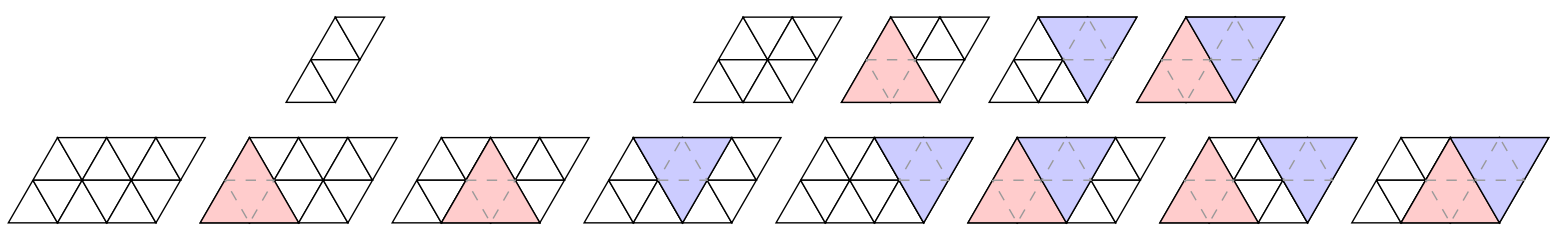

For $n \geqslant 4$, we note that we can group the tilings together in five groups based on the possible ends of the tilings. In particular, each tiling must end in one of the following five patterns:

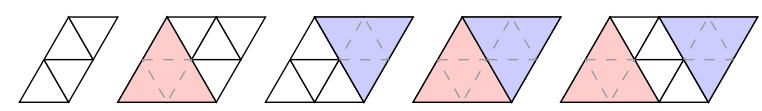

To complete the tilings, in the first group we have the number of ways to tile a $2 \times(n-1)$ board; in the next three groups we have the number of ways to tile a $2 \times(n-2)$ board; and in the final group we have the number of ways to tile the $2 \times(n-3)$ board. Combining these together gives the desired recursion. 
Using the recurrence and initial conditions, we can obtain the first few terms of the sequence $I(n)$ as follows:

$1,4,8,21,49,120,288,697,1681,4060,9800,23661,57121,137904,332928, \ldots$

This is sequence A097076 in the OEIS [5], which has been studied before, but this gives the first combinatorial interpretation of the sequence.

In the rest of this paper we will further explore this tiling and this sequence and establish several properties using various methods (i.e., combinatorial proofs, generating functions, etc.). In Section 2 we will briefly discuss other possible boards that could have been tiled and show a connection with Pell numbers. In Section 3 we will establish various recursive properties and identities of the sequence. In Section 4 we will refine our counting of the tilings by the types of triangles used and establish several basic facts. We finally conclude with some comments and open problems in Section 5.

\section{Tiling other kinds of boards}

There are several different triangular boards that can be used and depending on which board we settle on we get slightly different (but closely related) sequences. In particular, there are essentially four "natural" boards that we could consider. namely:
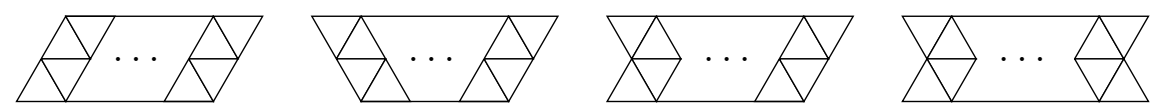

We will let $I(n), V(n), Y(n)$, and $X(n)$ denote the number of such tilings of these boards where $n$ is the length of the middle run. (The choice of notation comes from the shape of the board when $n=1$.)

We have already dealt with the sequence $I(n)$ in the previous section. Because the second and third boards have the same right hand edge, the recursion we found in Theorem 2 still applies and it only remains to find the first few terms needed to start the recursion (we leave finding these boards as an exercise to the reader). For the second board, i.e., the sequence $V(n)$, we get the following sequence:

$2,3,9,20,50,119,289,696,1682,4059,9801,23660,57122,137903,332929, \ldots$

This corresponds to sequence A097075 in the OEIS [5]. We note that this is closely related to the sequence for $I(n)$, as indicated in the following proposition.

Proposition 3. For $n \geqslant 1$, we have $I(n)=V(n)+(-1)^{n}$.

Proof. Triangles of type 1 in a tiling for the board for $V(n)$ or $I(n)$ will occur in groups of four, so for any tiling we find the first occurrence of one of these groups and perform one of the following operations wherein we flip the first part of the board: 

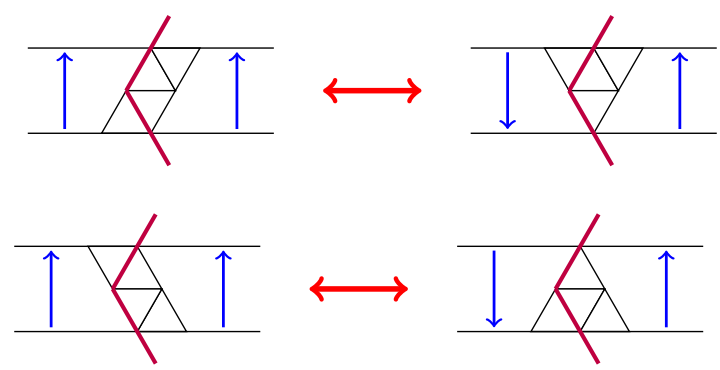

This is an invertible map between the first and second board that does not change the length of the middle run. Thus we have a near bijection between the two tilings. The only time this fails is if we have no triangles of type 1 available for us to apply this operation. For $n$ odd there is one such tiling for the second board (i.e., for the $V(n))$ and none for the first (i.e., for the $I(n)$ ). For $n$ even there is one such tiling for the first board (i.e., for the $I(n))$ and none for the second (i.e., for the $V(n)$ ). This establishes the result.

For the third board, i.e., the sequence $Y(n)$, we get the following sequence:

$2,5,12,29,70,169,408,985,2378,5741,13860,33461,80782,195025,470832, \ldots$

These are the Pell numbers (it is easily checked that the Pell numbers satisfy the recursion in Theorem 2 and the first few terms agree), which is sequence A000129 in the OEIS [5]. The tilings of this board provide a new combinatorial interpretation of the Pell numbers. (Note that previously a different interpretation involving tiling the $1 \times n$ rectangular strip with dominoes and two types of monomoes has been used to count Pell numbers and establish combinatorial properties, see [3].)

We can take the tilings of the third board and split them into two groups based on whether or not there is a $2^{-}$which uses the first small triangle on the upper left. If there is, then the remainder of the board to tile is a $2 \times(n-1)$ strip. If there is not, then the remainder of the board to tile is a $2 \times n$ strip. This proves the following.

Observation 4. For $n \geqslant 2$, we have $Y(n)=I(n-1)+I(n)$.

For the fourth board we cannot directly appeal to the recursion but we can group by what the end of the board looks like. The main difference in this case is that there are many ways to pull off the end of the board. We have the following where the term underneath indicates how many ways to tile the remainder of the board:
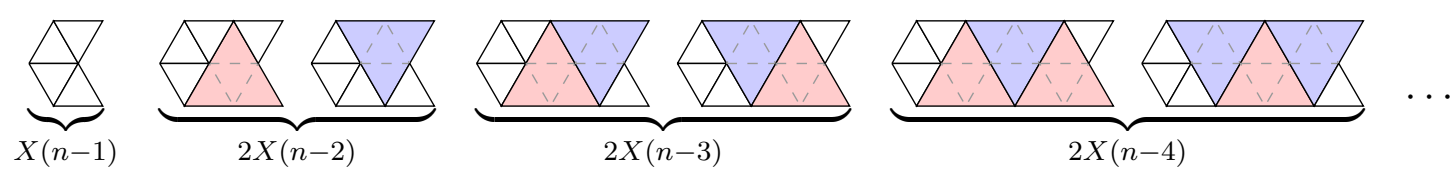

Therefore we can conclude

$$
X(n)=X(n-1)+2 X(n-2)+2 X(n-3)+\cdots+2 X(1)+2 .
$$


The final " +2 " on the end accounts for the two exceptional cases where we have alternating $2^{+}$and $2^{-}$triangles all the way across the board. Taking the difference of two consecutive terms we can conclude that the recurrence satisfies

$$
X(n)-X(n-1)=X(n-1)+X(n-2) \quad \text { or } \quad X(n)=2 X(n-1)+X(n-2) .
$$

Using the initial terms $1,3,7, \ldots$ we see that the number of such tilings is

$1,3,7,17,41,99,239,577,1393,3363,8119,19601,47321,114243,275807, \ldots$

This corresponds to the sequence A001333 in the OEIS [5]. This sequence has arisen frequently and tilings of this board gives a new combinatorial interpretation of these numbers.

\section{$3 \quad$ Recurrences and identities}

In this section we return to focusing on our sequence $I(n)$ and establish several properties. We will adopt the convention that $I(0)=1$ and let $I(x)=\sum_{j \geqslant 0} I(j) x^{j}$. From the recursion in Theorem 2 we can derive an expression for the generating function

$$
\begin{aligned}
\sum_{j \geqslant 3} I(j) x^{j} & =\sum_{j \geqslant 3} I(j-1) x^{j}+3 \sum_{j \geqslant 3} I(j-2) x^{j}+\sum_{j \geqslant 3} I(j-3) x^{j} \\
I(x)-4 x^{2}-x-1 & =x I(x)-x^{2}-x+3 x^{2} I(x)-3 x^{2}+x^{3} I(x) \\
I(x) & =\frac{1}{1-x-3 x^{2}-x^{3}}=\frac{1}{(1+x)\left(1-2 x-x^{2}\right)} .
\end{aligned}
$$

From this we can find an explicit expression for $I(n)$, namely

$$
I(n)=\frac{(1+\sqrt{2})^{n+1}}{4}+\frac{(1-\sqrt{2})^{n+1}}{4}+\frac{(-1)^{n}}{2} .
$$

We can use the generating function and the explicit solutions to establish some various properties of this sequence.

Proposition 5. The sequence $I(n)$ can be defined as $I(0)=I(1)=1$ and for $n \geqslant 2$

$$
I(n)=2 I(n-1)+I(n-2)+(-1)^{n} .
$$

This can be proved by showing that this recurrence produces the same generating function as given above, we omit the details here.

A more surprising result can be found by looking at the factorization of the first few terms.

\begin{tabular}{|c||c|c|c|c|c|}
\hline$I(4 k+i)$ & $k=0$ & $k=1$ & $k=2$ & $k=3$ & $k=4$ \\
\hline \hline$i=0$ & 1 & $3 \cdot 7$ & $17 \cdot 41$ & $99 \cdot 239$ & $577 \cdot 1393$ \\
\hline$i=1$ & $1^{2}$ & $7^{2}$ & $41^{2}$ & $239^{2}$ & $1393^{2}$ \\
\hline$i=2$ & $2 \cdot 1 \cdot 2$ & $2 \cdot 5 \cdot 12$ & $2 \cdot 29 \cdot 70$ & $2 \cdot 169 \cdot 408$ & $2 \cdot 985 \cdot 2378$ \\
\hline$i=3$ & $2 \cdot 2^{2}$ & $2 \cdot 12^{2}$ & $2 \cdot 70^{2}$ & $2 \cdot 408^{2}$ & $2 \cdot 2378^{2}$ \\
\hline
\end{tabular}


In particular the terms $\equiv 1(\bmod 4)$ appear to be perfect squares and the terms $\equiv 3$ (mod 4) appear to be twice perfect squares. In fact more can be said.

Theorem 6. Let $I(n)$ count the number of tilings of a $2 \times n$ triangular strip. Then for $n \geqslant 1$

$$
\begin{aligned}
I(4 m) & =(2 I(2 m-1)+1)(2 I(2 m)-1), \\
I(4 m+1) & =(2 I(2 m)-1)^{2}, \\
I(4 m+2) & =2(I(2 m-1)+I(2 m))(I(2 m)+I(2 m+1)), \\
I(4 m+3) & =2(I(2 m)+I(2 m+1))^{2} .
\end{aligned}
$$

Proof. The proof we give is computational based on $(1)$ and $(1+\sqrt{2})(1-\sqrt{2})=-1$. For the first two relationships we will use the following:

$$
\begin{aligned}
2 I(2 m)-1 & =2\left(\frac{(1+\sqrt{2})^{2 m+1}}{4}+\frac{(1-\sqrt{2})^{2 m+1}}{4}+\frac{1}{2}\right)-1 \\
& =\frac{(1+\sqrt{2})^{2 m+1}}{2}+\frac{(1-\sqrt{2})^{2 m+1}}{2}, \\
2 I(2 m-1)+1 & =2\left(\frac{(1+\sqrt{2})^{2 m}}{4}+\frac{(1-\sqrt{2})^{2 m}}{4}-\frac{1}{2}\right)+1 \\
& =\frac{(1+\sqrt{2})^{2 m}}{2}+\frac{(1-\sqrt{2})^{2 m}}{2} .
\end{aligned}
$$

For the first equation we have

$$
\begin{aligned}
(2 I(2 m-1)+1)(2 I(2 m)-1) \\
=\left(\frac{(1+\sqrt{2})^{2 m}}{2}+\frac{(1-\sqrt{2})^{2 m}}{2}\right)\left(\frac{(1+\sqrt{2})^{2 m+1}}{2}+\frac{(1-\sqrt{2})^{2 m+1}}{2}\right) \\
=\frac{(1+\sqrt{2})^{4 m+1}}{4}+\frac{(1-\sqrt{2})^{4 m+1}}{4}+\frac{1+\sqrt{2}}{4}+\frac{1-\sqrt{2}}{4} \\
=\frac{(1+\sqrt{2})^{4 m+1}}{4}+\frac{(1-\sqrt{2})^{4 m+1}}{4}+\frac{1}{2} \\
=I(4 m) .
\end{aligned}
$$

For the second equation we have

$$
\begin{aligned}
(2 I(2 m)-1)^{2} & =\left(\frac{(1+\sqrt{2})^{2 m+1}}{2}+\frac{(1-\sqrt{2})^{2 m+1}}{2}\right)^{2} \\
& =\left(\frac{(1+\sqrt{2})^{2 m+1}}{2}\right)^{2}+\left(\frac{(1-\sqrt{2})^{2 m+1}}{2}\right)^{2}-\frac{1}{2} \\
& =\frac{(1+\sqrt{2})^{4 m+2}}{4}+\frac{(1-\sqrt{2})^{4 m+2}}{4}-\frac{1}{2} \\
& =I(4 m+1) .
\end{aligned}
$$


For the third and fourth equations we will need the following:

$$
\begin{aligned}
I(2 m)+I(2 m-1) & =\frac{(1+\sqrt{2})^{2 m+1}}{4}+\frac{(1-\sqrt{2})^{2 m+1}}{4}+\frac{(1+\sqrt{2})^{2 m}}{4}+\frac{(1-\sqrt{2})^{2 m}}{4} \\
& =\sqrt{2}\left(\frac{(1+\sqrt{2})^{2 m+1}}{4}-\frac{(1-\sqrt{2})^{2 m+1}}{4}\right) \\
I(2 m)+I(2 m+1) & =\frac{(1+\sqrt{2})^{2 m+1}}{4}+\frac{(1-\sqrt{2})^{2 m+1}}{4}+\frac{(1+\sqrt{2})^{2 m+2}}{4}+\frac{(1-\sqrt{2})^{2 m+2}}{4} \\
& =\sqrt{2}\left(\frac{(1+\sqrt{2})^{2 m+2}}{4}-\frac{(1-\sqrt{2})^{2 m+2}}{4}\right) .
\end{aligned}
$$

For the third equation we have

$$
\begin{aligned}
2(I(2 m) & +I(2 m-1))(I(2 m)+I(2 m+1)) \\
& =4\left(\frac{(1+\sqrt{2})^{2 m+1}}{4}-\frac{(1-\sqrt{2})^{2 m+1}}{4}\right)\left(\frac{(1+\sqrt{2})^{2 m+2}}{4}-\frac{(1-\sqrt{2})^{2 m+2}}{4}\right) \\
& =\frac{(1+\sqrt{2})^{4 m+3}}{4}+\frac{(1-\sqrt{2})^{4 m+3}}{4}+\frac{1}{2} \\
& =I(4 m+2) .
\end{aligned}
$$

Finally, for the fourth equation we have

$$
\begin{aligned}
2(I(2 m)+I(2 m+1))^{2} & =2\left(\sqrt{2}\left(\frac{(1+\sqrt{2})^{2 m+2}}{4}-\frac{(1-\sqrt{2})^{2 m+2}}{4}\right)\right)^{2} \\
& =4\left(\frac{(1+\sqrt{2})^{4 m+4}}{16}+\frac{(1-\sqrt{2})^{4 m+4}}{16}-\frac{1}{8}\right) \\
& =\frac{(1+\sqrt{2})^{4 m+4}}{4}+\frac{(1-\sqrt{2})^{4 m+4}}{4}-\frac{1}{2} \\
& =I(4 m+3) .
\end{aligned}
$$

The preceding two results show some interesting properties of the sequence, but they do not directly appeal to the interpretation of tiling the strip with triangles. We can however give several results which have combinatorial proofs which do use tilings on the strip. These are analogous to results for Fibonacci numbers.

Theorem 7. If $I(n)$ is the number of ways to tile $a 2 \times n$ triangular strip and $m, n \geqslant 2$, then

$$
I(m+n)=I(m) I(n)+3 I(m-1) I(n-1)+I(m-1) I(n-2)+I(m-2) I(n-1) .
$$

Proof. The left hand side of this equation counts the number of ways to tile a $2 \times(m+$ $n)$ triangular strip. We now break the tilings up into six different groups which are determined by what happens at the dividing line after the $m$ th column of triangles. In particular we have one of the following possibilities: 

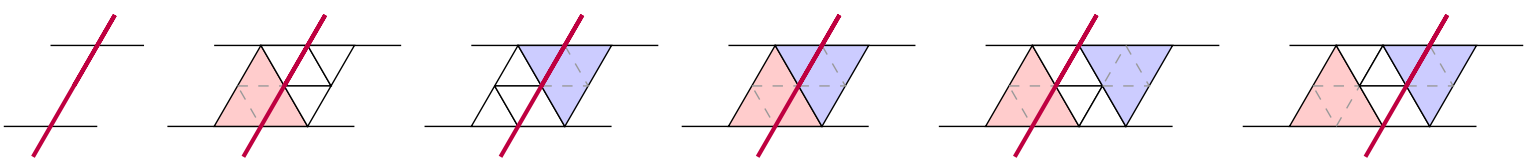

In the first case there is a clean break, so we have a $2 \times m$ strip on the left and a $2 \times n$ strip on the right remaining to be tiled and so this can happen in $I(m) I(n)$ different ways. In the next three cases we have a $2 \times(m-1)$ strip on the left and a $2 \times(n-1)$ strip on the right remaining to be tiled and so for each of these this can happen in $I(m-1) I(n-1)$ ways. In the fifth case we have a $2 \times(m-1)$ strip on the left and a $2 \times(n-2)$ strip on the right and this can happen in $I(m-1) I(n-2)$ ways. In the sixth case we have a $2 \times(m-2)$ strip on the left and a $2 \times(n-1)$ strip on the right and this can happen in $I(m-2) I(n-1)$ ways. Combining everything together yields the result.

Not only can we prove results analogous to the Fibonacci numbers using our interpretation, but thanks to Observation 1 we can also use the Fibonacci numbers themselves to help describe our new sequence.

Theorem 8. Let $F(n)$ be the Fibonacci numbers and let $I(n)$ be the number of ways to tile a $2 \times n$ triangular strip. Then

$$
I(n)=F(n+1)+\sum_{i=0}^{n-2} I(i) F(n+1-i) .
$$

Proof. By Observation 1 the number of tilings with no $2^{+}$triangle is $F(n+1)$.

Now consider the collection of remaining tilings and group them together by the location of the last $2^{+}$triangle, as in the following picture.

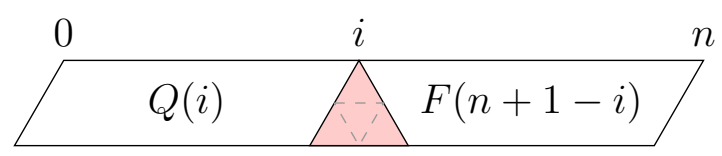

To the left of the last $2^{+}$triangle is a tiling of a $2 \times i$ strip for $0 \leqslant i \leqslant n-2$ which can be tiled in $I(i)$ ways. To the right is a trapezoidal region which only can contain $1^{+}, 1^{-}$and $2^{-}$triangles which by an analogous argument to Observation 1 can be tiled in $F(n+1-i)$ ways.

Finally, we give a combinatorial proof of an alternative recursion for the sequence $I(n)$.

Theorem 9. Let $I(n)$ be the number of ways to tile a triangular strip. Then for $n \geqslant 0$

$$
I(n+2)-1=4\left(\sum_{k=0}^{n} I(k)\right)-I(n) .
$$


Proof. The left hand side counts the number of ways to tile the $2 \times(n+2)$ strip where at least one $2^{+}$or $2^{-}$is used. We now show that the right hand side counts the same thing.

Similar to our last result we consider the location of the last large triangle. There are two possibilities. The first is that the last large triangle is a $2^{+}$(i.e., as in the illustration for the previous theorem). In this case there will be $I(i)$ tilings to the left of the triangle for $0 \leqslant i \leqslant n$ and 1 tiling to the right (i.e., it only involves $1^{+}$and $1^{-}$triangles). This gives a contribution of

$$
I(0)+I(1)+\cdots+I(n) .
$$

The other possibility is that the last large triangle is a $2^{-}$. In this case we want to consider what can come right before that, and there are three possibilities.

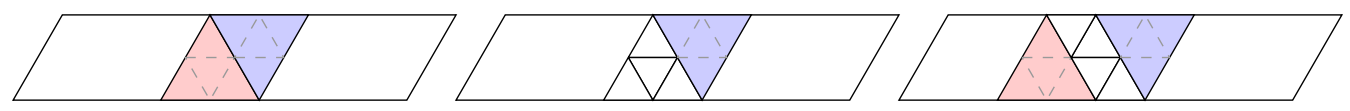

In the first two cases we are left with a tiling of the $I(i)$ board for $0 \leqslant i \leqslant n$. In the third case we are left with a tiling of the $I(i)$ board for $0 \leqslant i \leqslant n-1$. Therefore the contribution from this case is

$$
3 I(0)+3 I(1)+\cdots+3 I(n-1)+2 I(n) .
$$

Combining the two possibilities for the last type of large triangle now gives the result.

\section{A refined counting of the tilings}

So far we have only been concerned with the total number of tilings, but we can also refine our count by considering how many of each type of large triangle is used. In particular we will let $I(n, i, j)$ be the number of tilings of the $2 \times n$ triangular strip with $i$ of the $2^{+}$triangles and $j$ of the $2^{-}$triangles. We have some obvious symmetries, for example $I(n, i, j)=I(n, j, i)$ (since we can rotate any tiling by $180^{\circ}$ and $2^{+}$triangles become $2^{-}$ triangles and vice-versa).

We also have a recursion similar to that in Theorem 2

$$
\begin{aligned}
I(n, i, j)=I(n-1, i, j)+I(n-2, i- & 1, j)+I(n-2, i, j-1) \\
& +I(n-2, i-1, j-1)+I(n-3, i-1, j-1),
\end{aligned}
$$

which is based on examining the five endings and seeing how each one effects the length and the number of each type of large triangle.

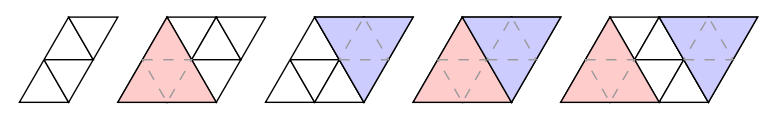

If we add the initial conditions $I(n, i, j)=0$ if $n<0, i<0$, or $j<0$ along with $I(0,0,0)=1$ and $I(0, i, j)=0$ otherwise, then this completely determines $I(n, i, j)$. 
We can also define a multi-variable generating function. Let

$$
\mathcal{I}(x, p, q)=\sum_{n, i, j} I(n, i, j) x^{n} p^{i} q^{j} .
$$

Then the above recursion along with the initial conditions give the following result

$$
\mathcal{I}(x, p, q)=\frac{1}{1-x-(p+q+p q) x^{2}-p q x^{3}} .
$$

Note this reduces to our previously found generating function when $p=q=1$.

We will be interested in examining what happens when we fix the number of certain types of triangles. To start we will list the value of these statistics for $2 \leqslant n \leqslant 9$ in Table 1.

Table 1: Values of $I(n, i, j)$ for $2 \leqslant n \leqslant 9$.

\begin{tabular}{|c||c|c|}
\hline$I(2, i, j)$ & $j=0$ & $j=1$ \\
\hline \hline$i=0$ & 1 & 1 \\
\hline$i=1$ & 1 & 1 \\
\hline
\end{tabular}

\begin{tabular}{|c||c|c|}
\hline$I(3, i, j)$ & $j=0$ & $j=1$ \\
\hline \hline$i=0$ & 1 & 2 \\
\hline$i=1$ & 2 & 3 \\
\hline
\end{tabular}

\begin{tabular}{|c||c|c|c|}
\hline$I(4, i, j)$ & $j=0$ & $j=1$ & $j=2$ \\
\hline \hline$i=0$ & 1 & 3 & 1 \\
\hline$i=1$ & 3 & 7 & 2 \\
\hline$i=2$ & 1 & 2 & 1 \\
\hline
\end{tabular}

\begin{tabular}{|c||c|c|c|}
\hline$I(5, i, j)$ & $j=0$ & $j=1$ & $j=2$ \\
\hline \hline$i=0$ & 1 & 4 & 3 \\
\hline$i=1$ & 4 & 13 & 8 \\
\hline$i=2$ & 3 & 8 & 5 \\
\hline
\end{tabular}

\begin{tabular}{|c||c|c|c|c|}
\hline$I(6, i, j)$ & $j=0$ & $j=1$ & $j=2$ & $j=3$ \\
\hline \hline$i=0$ & 1 & 5 & 6 & 1 \\
\hline$i=1$ & 5 & 21 & 21 & 3 \\
\hline$i=2$ & 6 & 21 & 19 & 3 \\
\hline$i=3$ & 1 & 3 & 3 & 1 \\
\hline
\end{tabular}

\begin{tabular}{|c||c|c|c|c|}
\hline$I(7, i, j)$ & $j=0$ & $j=1$ & $j=2$ & $j=3$ \\
\hline \hline$i=0$ & 1 & 6 & 10 & 4 \\
\hline$i=1$ & 6 & 31 & 44 & 15 \\
\hline$i=2$ & 10 & 44 & 55 & 18 \\
\hline$i=3$ & 4 & 15 & 18 & 7 \\
\hline
\end{tabular}

\begin{tabular}{|c||c|c|c|c|c|}
\hline$I(8, i, j)$ & $j=0$ & $j=1$ & $j=2$ & $j=3$ & $j=4$ \\
\hline \hline$i=0$ & 1 & 7 & 15 & 10 & 1 \\
\hline$i=1$ & 7 & 43 & 80 & 46 & 4 \\
\hline$i=2$ & 15 & 80 & 131 & 69 & 6 \\
\hline$i=3$ & 10 & 46 & 69 & 37 & 4 \\
\hline$i=4$ & 1 & 4 & 6 & 4 & 1 \\
\hline
\end{tabular}

\begin{tabular}{|c||c|c|c|c|c|}
\hline$I(9, i, j)$ & $j=0$ & $j=1$ & $j=2$ & $j=3$ & $j=4$ \\
\hline \hline$i=0$ & 1 & 8 & 21 & 20 & 5 \\
\hline$i=1$ & 8 & 57 & 132 & 110 & 24 \\
\hline$i=2$ & 21 & 132 & 271 & 204 & 42 \\
\hline$i=3$ & 20 & 110 & 204 & 147 & 32 \\
\hline$i=4$ & 5 & 24 & 42 & 32 & 9 \\
\hline
\end{tabular}

There are many interesting properties that can be seen in these arrays. For example the first rows correspond to when we only have one type of large triangle which by Observation 1 relates to the Fibonacci numbers. And indeed the first rows correspond to the coefficients of the Fibonacci polynomials (see sequence A011973 in the OEIS [5]). 
The last rows also follow a nice pattern.

Proposition 10. Let $I(n, i, j)$ be defined as above. Then

$$
\begin{aligned}
I(2 n, n, j) & =\left(\begin{array}{l}
n \\
j
\end{array}\right), \\
I(2 n+1, n, j) & =(n+j+1)\left(\begin{array}{l}
n \\
j
\end{array}\right) .
\end{aligned}
$$

Proof. For the case of $I(2 n, n, j)$ we note that there are a full set of $2^{+}$triangles, such as in the following picture when $2 n=6$.

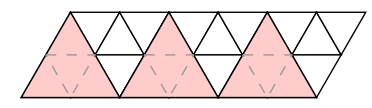

This leaves precisely $n$ holes for the $j$ triangles of type $2^{-}$to place in $I(2 n, n, j)$, and there are $\left(\begin{array}{l}n \\ j\end{array}\right)$ ways to pick which holes will be used.

For the case $I(2 n+1, n, j)$ we subdivide the count into three groups, illustrated below for $n=4, j=2$.

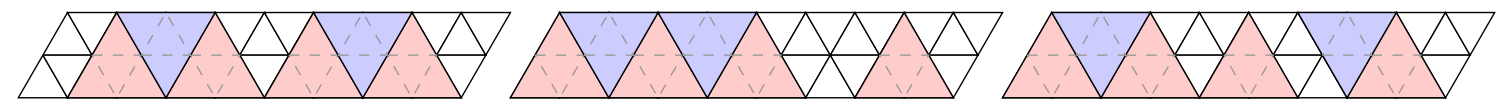

In the first group are arrangements where the $2^{+}$leave a gap at the very beginning that can't be filled, as before there are $\left(\begin{array}{c}n \\ j\end{array}\right)$ ways to put in $j$ of the $2^{-}$type triangles. The second group are arrangements where the extra gap is in the middle or end but does not contain a $2^{-}$triangle, this leaves us with $n-1$ other gaps available and so there are $n\left(\begin{array}{c}n-1 \\ j\end{array}\right)$ (the factor $n$ comes from choosing the location of the gap). The third group are arrangements where the extra gap is in the middle or end and does contain a $2^{-}$triangle, this leaves us with $n-1$ other gaps to put the $j-1$ remaining triangles so there are $2 n\left(\begin{array}{c}n-1 \\ j-1\end{array}\right)$ (the 2 comes from the two ways to fill the gap). Combining we have

$$
\left(\begin{array}{l}
n \\
j
\end{array}\right)+n\left(\begin{array}{c}
n-1 \\
j
\end{array}\right)+2 n\left(\begin{array}{c}
n-1 \\
j-1
\end{array}\right)=(n+j+1)\left(\begin{array}{l}
n \\
j
\end{array}\right) .
$$

We have seen that the first row sums correspond to Fibonacci numbers so we might see if a similar pattern exists for other row sums. In Table 2 is a summary of the row sum data from Table 1. In particular, the second row sums have also appeared before as sequence A067331 in the OEIS [5] as the convolution of $F(n+1)$ and $F(n+3)$. More generally, we have the following result.

Proposition 11. Let $I(n, i, j)$ be defined as above. Then

$$
\sum_{j} I(n, i, j)=\sum_{\substack{\ell_{1} \geqslant 0 \\ \ell_{2}, \ldots, \ell_{i+1} \geqslant 2 \\ \ell_{1}+\ell_{2}+\cdots+\ell_{i+1}=n}}\left(\prod_{k=1}^{i+1} F\left(\ell_{k}+1\right)\right) .
$$


Table 2: Values of $\sum_{j} I(n, i, j)$.

\begin{tabular}{c|ccccc}
$\sum_{j} I(n, i, j)$ & $i=0$ & $i=1$ & $i=2$ & $i=3$ & $i=4$ \\
\hline$n=0$ & 1 & & & & \\
$n=1$ & 1 & & & & \\
$n=2$ & 2 & 2 & & & \\
$n=3$ & 3 & 5 & & & \\
$n=4$ & 5 & 12 & 4 & & \\
$n=5$ & 8 & 25 & 16 & & \\
$n=6$ & 13 & 50 & 49 & 8 & \\
$n=7$ & 21 & 96 & 127 & 44 & \\
$n=8$ & 34 & 180 & 301 & 166 & 16 \\
$n=9$ & 55 & 331 & 670 & 513 & 112
\end{tabular}

Proof. This boils down to using Observation 1 and noting that after we place the $2^{+}$ triangles that the gaps between can be tiled arbitrarily with $2^{-}$triangles. In particular the $\ell_{k}$ are measuring the sizes of the gaps (with regard to the top row), such as in the following picture.

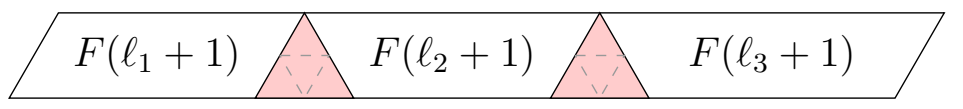

Therefore once we know the sizes of the gaps to fill we can know how many ways there are to place the $2^{-}$triangles in this configuration. The first gap $\ell_{1}$ can be any size, and the remaining gaps must have size at least 2 . The result now follows.

Row sums fix the number of $2^{+}$triangles we could use in a tiling. We could alternatively take "anti-diagonal" sums and get the number of tilings where the number of large triangles is fixed, i.e., consider

$$
\sum_{i+j=m} I(n, i, j)
$$

The first few values of these sums are given in Table 3. Remarkably, this entire table has already appeared as sequence A217874 in the OEIS [5], but in a completely different setting. Namely this is related to the figurate numbers for the $n$-dimensional cross polytope (see [2]). We give a direct count for these values.

Theorem 12. Let $I(n, i, j)$ be as defined above and consider the number of tilings using exactly $m$ large triangles. Then

$$
\sum_{i+j=m} I(n, i, j)=\sum_{k}\left(\begin{array}{c}
m+1 \\
2 k+1
\end{array}\right)\left(\begin{array}{c}
n-m+2 k \\
m
\end{array}\right) .
$$


Table 3: Values of $\sum_{i+j=m} I(n, i, j)$.

\begin{tabular}{c|ccccccccc}
$\sum_{i+j=m} I(n, i, j)$ & $m=0$ & $m=1$ & $m=2$ & $m=3$ & $m=4$ & $m=5$ & $m=6$ & $m=7$ & $m=8$ \\
\hline$n=0$ & 1 & & & & & & & \\
$n=1$ & 1 & & & & & & & \\
$n=2$ & 1 & 2 & 1 & & & & & \\
$n=3$ & 1 & 4 & 3 & & & & & & \\
$n=4$ & 1 & 6 & 9 & 4 & 1 & & & & \\
$n=5$ & 1 & 8 & 19 & 16 & 5 & & & & \\
$n=6$ & 1 & 10 & 33 & 44 & 25 & 6 & 1 & & \\
$n=7$ & 1 & 12 & 51 & 96 & 85 & 36 & 7 & & \\
$n=8$ & 1 & 14 & 73 & 180 & 225 & 146 & 49 & 8 & 1 \\
$n=9$ & 1 & 16 & 99 & 304 & 501 & 456 & 231 & 64 & 9
\end{tabular}

The key to the proof will be in giving another interpretation of these tilings. In particular for every such tiling we can associate it with a sequence where the values indicate the gaps in the placements. In particular, when the gap forms a parallelogram we will associate it with the length of the middle segment between the two; and when the gap is a trapezoid then we will associate it with the negative of the length of the middle segment between the two. Note the last gap term is superfluous and will be dropped. An example is shown below for $n=20$ and the sequence $(3,1,-2,0,-5,-2)$.

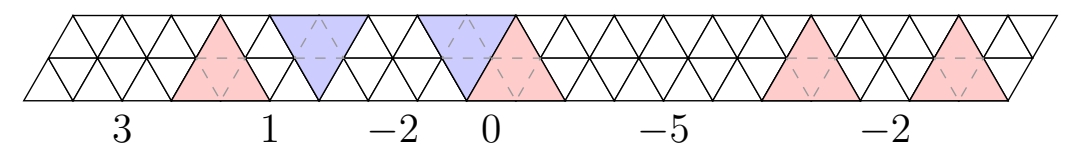

The problem now boils down to understanding what sequences are possible, In particular, we will group them by sign changes and determine the number inside of each group via a "bars and stars" argument. To help illustrate the proof we demonstrate what is possible for $n=5$ and $m=2$ in Figure 1.

Proof of Theorem 12. We need to determine two things, how to group patterns together, and for a given group of patterns how many tilings are possible.

For the groups we will read off the types of triangles from left to right where we associate the left edge or a $2^{-}$with a "-", and the right edge or a $2^{+}$with a "+". As an example, for $n=5$ and $m=2$ our sign patterns are the following:

\begin{tabular}{|c|l|}
\hline sign changes & sign patterns \\
\hline 1 & $(-,+,+,+),(-,-,+,+),(-,-,-,+)$ \\
\hline 3 & $(-,+,-,+)$ \\
\hline
\end{tabular}

Finally we will group together all tilings whose patterns have the same number of changes in their sign pattern when read from left to right. Since we start and end with different 


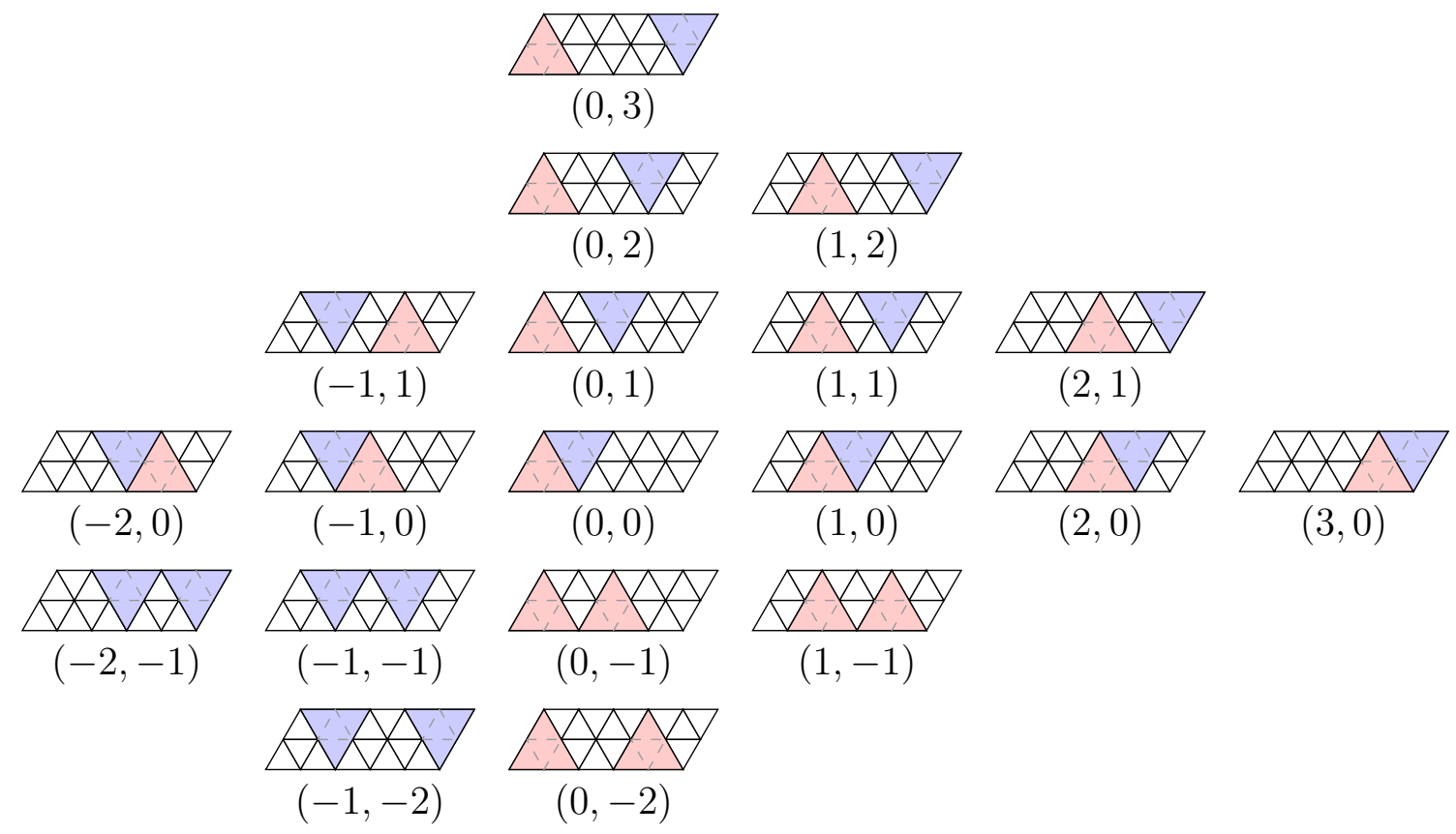

Figure 1: All tilings of $2 \times 5$ strip with two large triangles.

signs the number of changes is odd and we denote this by $2 k+1$. There are $m+2$ signs, and so there are $m+1$ possible indices $i$ in which the $i$-th and $(i+1)$-st signs can differ, which implies the number of patterns with $2 k+1$ sign changes is $\left(\begin{array}{c}m+1 \\ 2 k+1\end{array}\right)$.

Given any sign pattern we need to determine the number of tilings associated with that pattern. To do this we first pack the pattern tightly starting with the left. Each time there is a sign change we can pack tightly with no gap, but when there is no sign change we will have four triangles needed to fill the gap. In particular we have the following:

$$
\text { extra columns }=\frac{4 n-4 m-4(m+1-(\# \text { sign changes }))}{4},
$$

where extra columns indicates how many columns are not covered by part of a large triangle, i.e., how much we have available to rearrange. On the right hand side we start with $4 n$ small triangles total, take off 4 triangles for each large triangle in the packing, and then take off 4 for every time a sign did not change (i.e., there are $m+1$ possible sign changes and we take off the sign changes). If we let $2 k+1$ be the number of sign changes we have

$$
\text { extra columns }=n-2 m+2 k .
$$

If this is negative then we cannot even pack the triangles in as tightly as possible and so there are 0 packings associated with the pattern. On the other hand if this is non-negative then we can distribute the extra columns that we have among the $m+1$ different gaps between the triangles. This can be done in

$$
\left(\begin{array}{c}
(n-2 m+2 k)+(m+1)-1 \\
(m+1)-1
\end{array}\right)=\left(\begin{array}{c}
n-m+2 k \\
m
\end{array}\right)
$$


different ways.

Finally we combine the two results together to get the desired formula.

\section{Conclusion}

In this paper we have looked at ways to tile the triangular strip. We have seen that this has connections with the Fibonacci numbers and that the resulting sequence of numbers has many beautiful recurrences and properties. There are still a number of avenues left to explore and we mention several of these here.

- We saw that related tilings could be associated with the Pell numbers and with sequence A001333 in the OEIS [5]. It would be interesting to explore combinatorial proofs using these tilings to give new identities and properties of these sequences.

- In Proposition 5 and Theorem 6 we established some properties of the sequence $I(n)$ but did not use combinatorial proofs for these results. A combinatorial explanation has been subsequently found by Shattuck and will appear in a forthcoming paper [4].

- One could consider larger triangular boards and with larger possible sets of triangles and ask for the number of tilings.

We look forward to seeing more progress on the above questions and related problems in future work.

\section{Acknowledgment}

The authors thank the anonymous referee for their careful reading of the paper and many useful suggestions for improving the exposition.

\section{References}

[1] Arthur Benjamin and Jennifer Quinn. Proofs that Really Count: The Art of Combinatorial Proof. MAA, 2003.

[2] Hyun Kwang Kim. On regular polytope numbers. Proceedings of the American Mathematical Society, 131:65-75, 2002.

[3] Mark A. Shattuck. Tiling proofs of some formulas for the Pell numbers of odd index. Integers 9:53-64, 2009.

[4] Mark A. Shattuck. Combinatorial proofs of some formulas related to triangular tilings. Preprint.

[5] Neil Sloane. The On-Line Enyclopedia of Integer Sequences, www. oeis.org. 